normal folded TYR

\section{Mutant protein in Siamese cats may be involved in neurodegenerative disorders}

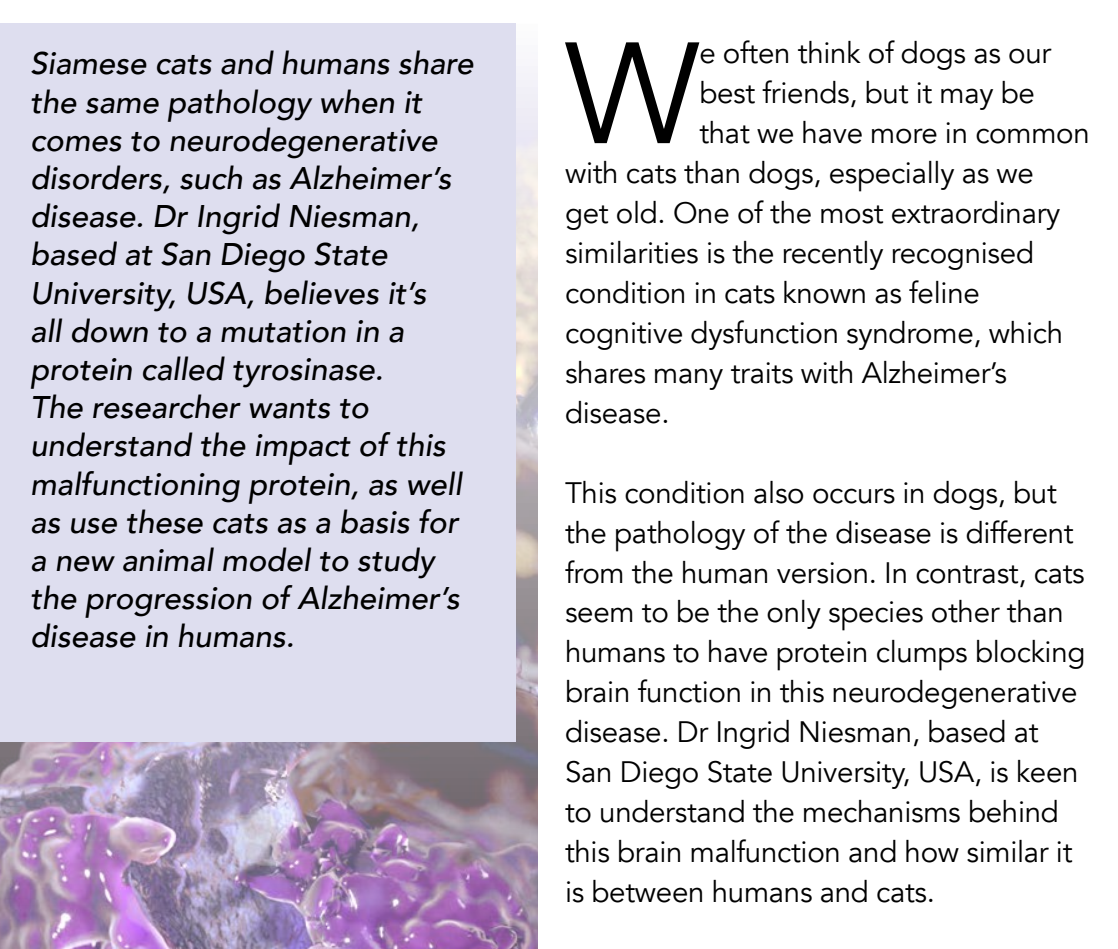

work above $39^{\circ} \mathrm{C}$. This difference explains why the cat's warm parts of the body have hair with no melanin - white cream hair - whereas, in cooler areas, this enzyme is active and can produce Incredibly this the hair a dark colour. one amino acid in the protein but has a dramatic effect on its function.

\section{IT'S NOT ALL ABOUT THE FUR} Fur and brain seem incredibly disconnected, but in this case, are very much linked by the protein tyrosinase. surprising as the cells that generate the skin and some brain cells originate from the same group in the embryo. This means that if tyrosinase doesn't work in warm areas of Siamese skin, then the protein also won't function in brain cells. This lack of function may look cute as different colours for Siamese cats, brain, where tyrosinase is involved in producing dopamine. When it malfunctions, this protein forms clumps in the brain and causes symptoms that are very similar to Alzheimer's in humans. In cats, this condition is characterised by altered sleeping and eating patterns, disorientation, loss of house training, and excessive nighttime vocalisations. until the final stages of the disease that the condition can be confirmed.

Dr Niesman knows that there has been very little research on this subject but is keen to raise awareness about this a few decades ago, and anima behaviourists are designing interventions
for pet owners", says the researcher. patient's quality

Their working hypothesis is that repeated stress events lead to neurodegenerative the cooler parts of the body (essentially a lighter colour. In 2005, a group of California found that Siamese cats have a mutation in a protein called tyrosinase. This mutated protein is involved in producing the pigment melanin at Diagnosis is challenging, and it's not condition. "The disease was recognised

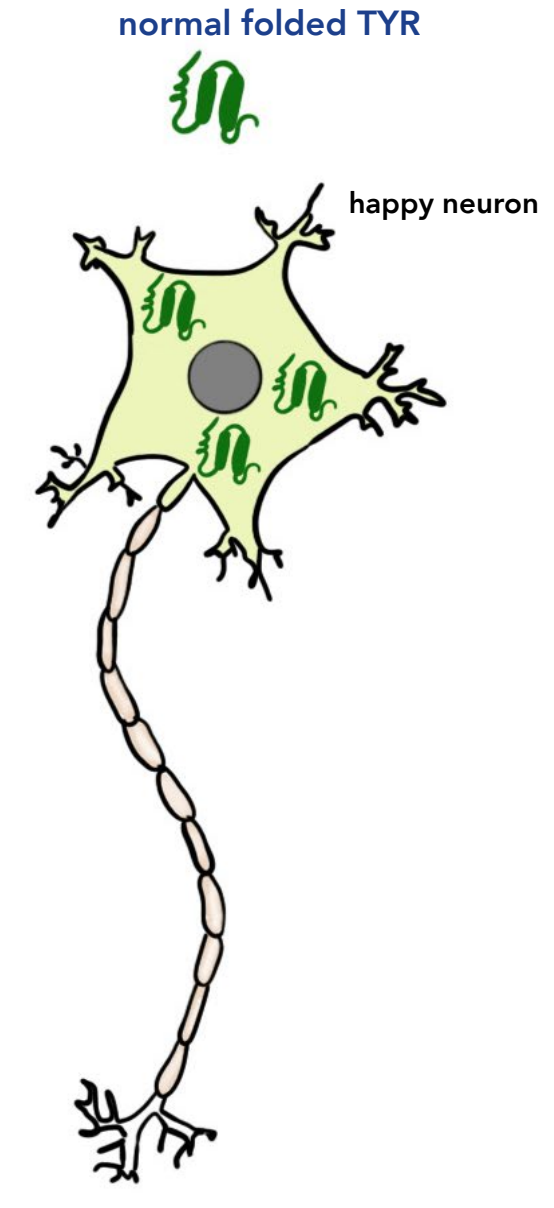

misfolded mutant TYR

aggregated mutant TYR

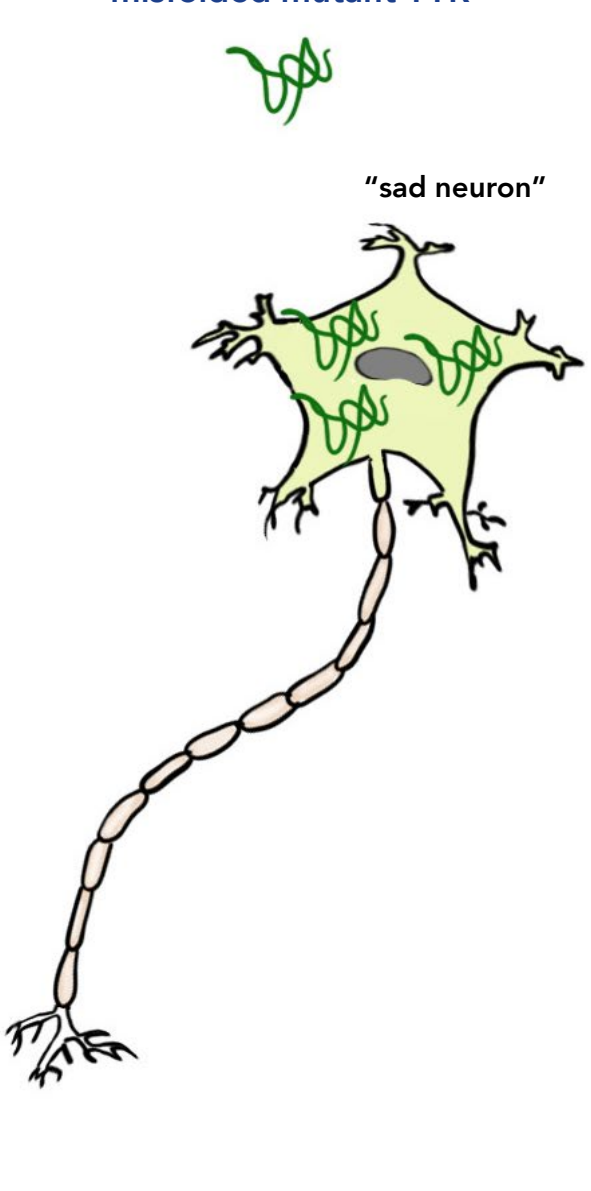

Pas dying neuron

Schematic of a normal, misfolded mutant and aggregated mutant neuron.

"Yet, the outcome for either dogs or cats step in this process involves a boost is the same - eventual euthanasia." in the production of tyrosinase, which over time can become quite

The problem is that clinical diagnosis requires an MRl, which is an expensive test many cat owners will not consider for their ageing cats. However, Dr Niesman and herteam believe they have problematic if it is the faulty version of this protein. Dr Niesman believes this the first attempt to look at this using a cell biology app

screning tol to detect he condition early. This way, veterinarins may have time to implement special dietary supplements or create environmental enrichment protocols to improve their

\section{IS IT TOXIC FOR CELLS?} Before attempting to explain any determine if the mutant form of tyrosinase is toxic for cells. Dr N

More research is needed, but it all seems to point to the fact that conditions in

Siamese cats, in a mechanism that may be related to the mutant tyrosinase present in brain cells. His well known that under stress, animals need to
generate more dopamine. The first mutant Siamese tyrosinase is toxic for brain cells.

used a combination of molecular biology produce this protein. funding." and microscopy to figure out if Siamese tyrosinase is potentially harmful when

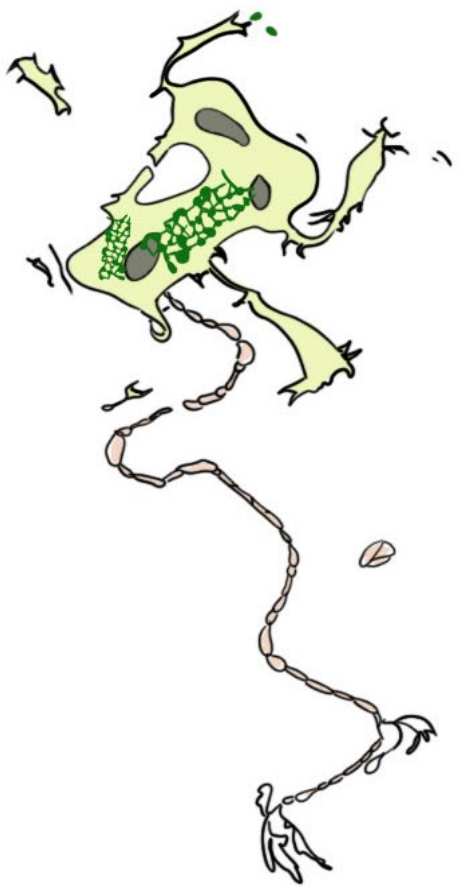

Using human cells (not brain cells), the team injected fluorescent forms of normal and mutant tyrosinase and found that mutant tyrosinase was degraded faster than its normal counterpart. When this degradation was blockedbody temperature $\left(40^{\circ} \mathrm{C}\right.$ in cats) the en found large clumps of protein accumulating in sensitive areas in the cells producing Siamese tyrosinase.

These clumps clog normal cell function can lead to cell death. More$$
\text { research is needed, }
$$
but it all seems to point to the fact that mutant Siamese
tyrosinase is toxic for brain cells. "We need longitudinal survey groups to track Slamese-breed cats over domestic short hair controls", says the researcher. "We need solid cat behavioural markers and people willing 


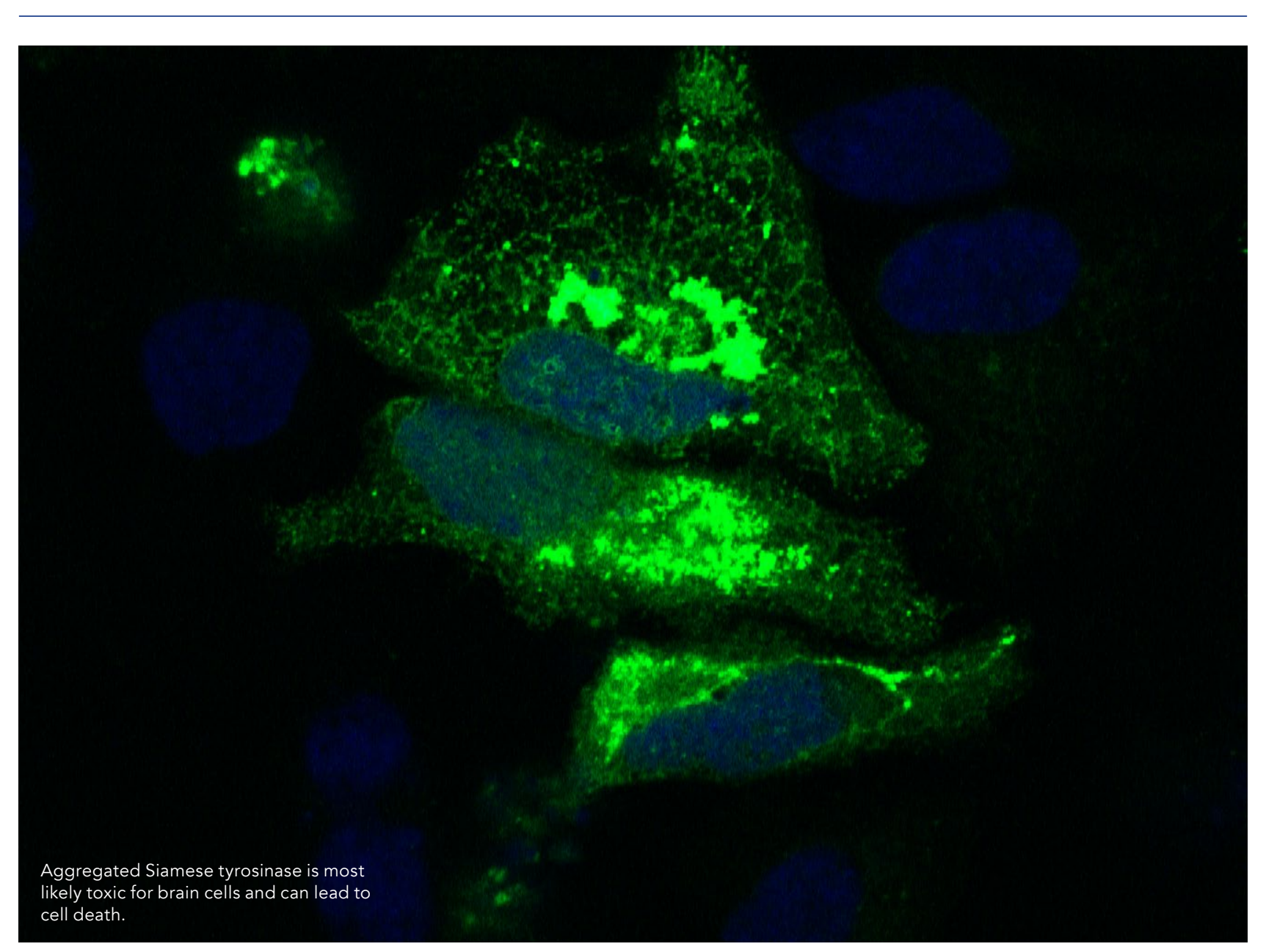

\section{THE SAME IN HUMANS} In humans, Alzheimer's disease can be diagnosed relatively early in its development. These patients often describe changes in smell and taste, or frequent memory lapses, such as forgetting words or the location of

new ways to counteract toxicity caused
by this faulty protein, which is involved in many neurological conditions.

LOOKING FOR A NEW MODEL With this mind, Dr Niesman and her

neurodegenerative conditions based on issues and symptoms. "We believe

Ideally, this model would involve an animal with a lifespan long enough to develop clear signs of Alzheimer's disease. This would allow researchers to study the mechanisms that trigger the development of this disease as

neurodegenerative conditions based on issues and symptoms. "We believe that such a mode damage to nerve it difficult for the person to perform person to perform
routine tasks, and routine tasks, and
some patients may show a tendency tow Dr Niesman believes that such a model, which has gone virtually unstudied for this purpose, might be Siamese cats.

which has gone virtually unstudied
for this purpose, might be Siamese cats", said Dr Niesman.

the mutant tyrosinase. While genetically As memory and cognitive skills continue modified mouse models have provided to deteriorate, individuals lose the ability to carry a conversation.

Curiously, humans also have a mutation in tyrosinase that functions in a similar way to Siamese cats. In this case, the mutant protein malfunctions at norma body temperature andit can become toxic for brain cells. For Dr N wesman, these feline firends have the potential to a great deal of information about believes the scientific community would benefit greatly from having a naturally occurring animal model to study the pathological features of neurological conditions in humans. Th model would be ideal for identifying some of the earliest neurological, behavioural and biochemical changes that occur dung the developm
Until recently, the use of cats as a potential model for human neurodegenerative diseases was considered farfetched. This is somewhat surprising because cats can show human-like neuropathological changes, suggesting some therapies may work both humans and cats. What's more, Dr Niesman believes cats don't have to be simply models for human condtions, but they can unlock can benefit both animats a

\section{(8) Behind the Research} Dr Ingrid Niesman

E: iniesman@sdsu.edu T: +16195944523 in www.linkedin.com/in/Ingrid-niesman-ms-phd-70aa8629

\section{Research Objectives}

Dr Niesman aims to unravel the relationships between feline colouration, behaviour and incresed

\section{Detail}

Ingrid Niesman

(5) MC 4614

San Diego, CA 92182

USA

Bio

Ingrid Niesman, MS, PhD is the Director of the SDSU EM Facility. She teaches TEM and SEM courses. Her cat-focused writing can be found on the website The Conscious Cat and in other national magazines. She is a 2019 MUSE award winner for her series on feline infectious peritonitis.

\section{Collaborators}

- Chris Glembotski

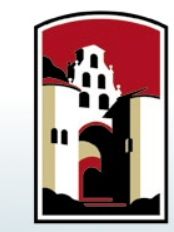

SAN DIEGO STATE UNIVERSITY

\section{References}

Niesman, I. (2020). Siamese cat tyrosinase has enhanced proteasome degradation and increased cellular aggregation. BioRxiv. Available at: https://doi. org/10.1101/2020.06.03.132613 [Preprint]

\section{Personal Response}

What is the next step to create an animal model to

II PSC - induced pluripotent stem cells - are the gold standard for developing models of neurodegenerative but feline cells are not. A major goal for me is to tap into the SoCal stem cell community for expertise in developing pluripotent lines for gene editing and differentiation into feline neurons. Disease-in-a-dish models are an excellent starting point to screen for

Another critical goal is initiation of a longitudinal survey of aging Siamese and non-pedigreed cats to assess behaviour and pathology ultimately correlate. 